\title{
Life-Threatening Intracranial Hypotension after Skull Base Surgery with Lumbar Drainage
}

\author{
Seiichiro Hirono ${ }^{1}$ Daisuke Kawauchi ${ }^{1}$ Yoshinori Higuchi ${ }^{1}$ Taiki Setoguchi ${ }^{1}$ Kazunori Kihara ${ }^{2}$ \\ Kentaro Horiguchi ${ }^{1}$ Ken Kado ${ }^{2}$ Motoki Sato ${ }^{2}$ Kazumasa Fukuda ${ }^{2}$ Takao Nakamura ${ }^{2}$ \\ Naokatsu Saeki ${ }^{1}$ Iwao Yamakami ${ }^{2}$
}

1 Department of Neurological Surgery, Chiba University Graduate School of Medicine, Chuoku, Chiba, Japan

2 Department of Neurosurgery, Chiba Central Medical Center,

Wakabaku, Chiba, Japan

\author{
Address for correspondence Seiichiro Hirono, MD, PhD, Department \\ of Neurological Surgery, Chiba University Graduate School of \\ Medicine, 1-8-1 Inohana, Chuoku, Chiba 260-8670, Japan \\ (e-mail: s-hirono@umin.ac.jp).
}

J Neurol Surg Rep 2015;76:e83-e86.

\begin{abstract}
Keywords

- intracranial hypotension

- skull base surgery

- lumbar drainage

- epidural blood patch

- midline shift

Although lumbar drainage (LD) is widely used in skull base surgery (SBS), no cases with intracranial hypotension (IH) following LD-assisted SBS have been reported, and skull base surgeons lack awareness of this potentially life-threatening condition. We report two cases of IH after LD-assisted SBS, a spheno-orbital meningioma and an osteosarcoma in the orbit. Despite a minimal amount of cerebrospinal fluid (CSF) drainage and early LD removal, severe postural headache and even a deteriorating consciousness level were observed in the early postoperative course. Neuroimages demonstrated epidural fluid collections, severe midline shift, and tonsillar sag compatible with $\mathrm{IH}$. Epidural blood patch (EBP) immediately and completely reversed the clinical and radiologic findings in both patients. IH should be included in the differential diagnosis of postural headache after LD-assisted SBS that can be managed successfully with EBP. Persistent leakage of CSF at the LD-inserted site leads to IH. Broad dural dissection and wide removal of bony structure may be involved in the midline shift. EBP should be performed soon after conservative management fails. Further reports will determine the risk factors for IH development following LD-assisted SBS.
\end{abstract}

\section{Introduction}

Lumbar puncture (LP) and lumbar drainage (LD) are widely used diagnostic procedures and also used in aneurysmal clipping and skull base surgeries for brain relaxation and better surgical manipulation. Among the complications associated with LP/LD is a cerebrospinal fluid (CSF) leak at the puncture site and subsequent intracranial hypotension $(\mathrm{IH})$, especially after clipping surgeries. ${ }^{1-3}$ It has been increasingly recognized as a cause of postural headache and other neurologic symptoms.

Neurosurgeons are interested in this rare but potentially life-threatening entity, especially because the mechanisms that cause them have not been elucidated, especially why most of the LD-induced IH cases occur after clipping surgeries. ${ }^{4}$ In addition, the risk factors for developing $\mathrm{IH}$ have not yet been clarified. Most of the reported cases following aneurysmal surgeries involve supratentorial aneurysms, ${ }^{2-5}$ and no cases following craniotomy for skull base tumor resection have been reported so far, which deepens the mystery about the pathophysiology of IH following LD-assisted craniotomy.

Here we report two cases of LD-induced $\mathrm{IH}$ following supratentorial skull base surgeries. These have great potential to help reveal the pathophysiology and risk factors associated with LD-induced IH after craniotomy. received

August 12, 2014 accepted after revision

December 16, 2014

published online

April 6, 2015
DOI http://dx.doi.org/

10.1055/s-0035-1547369. ISSN 2193-6366. (c) 2015 Georg Thieme Verlag KG
Stuttgart · New York

License terms

(®) (1) $\circledast$ 


\section{Case Report}

\section{Case 1}

A 57-year-old woman with a body mass index (BMI) of 17.0 underwent craniotomy for resection of a right spheno-orbital meningioma ( - Fig. 1A). LD was placed after the induction of general anesthesia to reduce CSF volume and provide brain relaxation. The frontotemporal craniotomy with an orbitozygomatic osteotomy was performed to expose the frontal and temporal dura and the periorbita because of orbital involvement of the tumor. The sphenoid wing and the bone around the superior orbital fissure were drilled extradurally under the microscope. The bone drilling was continued up to remove the roof of the bony orbit and the anterior half of the middle temporal fossa that leads to the wide detachment of the dura from the anterior and middle skull base. The dura was opened wide, and the lesion was resected both intradurally and extradurally. Because the tumor within the cavernous sinus was not removed aggressively, only a subtotal resection was achieved. During the reconstruction, the bone segments were reattached with titanium miniplates and screws. LD was removed at the end of the procedure 9 hours after insertion.

On postoperative day 1 when the patient was lying prone all day, she was alert and her neurologic examination showed no evidence of any focal signs. In the morning of postoperative day 2 when she was allowed to walk, she became lethargic and then rapidly became comatose with anisocoria within 1 hour. Her head computed tomography (CT) scan demonstrated a severe left to right shift and extradural fluid collection (-Fig. 1B). She underwent evacuation of the fluid and a decompressive craniectomy. However, neither her consciousness nor the severe midline shift improved on images (-Fig. 1C). A bedridden management thereafter improved both her consciousness and the midline shift on CT ( - Fig. 1D). She was again allowed to walk 5 days after the second surgery, and her consciousness deteriorated again and anisocoria was observed immediately $(-$ Fig. 1E). Thereafter, she was kept bedridden for 8 days. Cranioplasty and a subsequent $15-\mathrm{mL}$ epidural blood patch (EBP) at 19 days after the initial craniotomy resolved her symptoms immediately and completely (-Fig. 1F).

\section{Case 2}

A 31-year-old man, whose BMI was 27.3 and a history of bilateral retinoblastoma at age 10 months, presented with headache and left temporal pain. Neuroimages revealed osteosarcoma at the lateral wall of the left orbit ( - Fig. 2A). LD was inserted preoperatively for facilitating brain retraction. Multiple puncture attempts with an $18 \mathrm{G}$ needle were needed to place the drain. The frontotemporal craniotomy and an orbitozygomatic osteotomy were performed, and the dura of the anterior and middle skull base was broadly dissected from its attachment. The lesion was totally removed en bloc with surrounding structures including most of the middle skull base bone, the superior and lateral wall of the orbit, and the temporal muscle. A total of $100 \mathrm{~mL}$ CSF was intermittently removed throughout the operation, and the drain was removed on the day following the surgery.

Although the postoperative course was uneventful, the patient experienced a severe postural headache, especially in the posterior cervical region, which required bed rest all
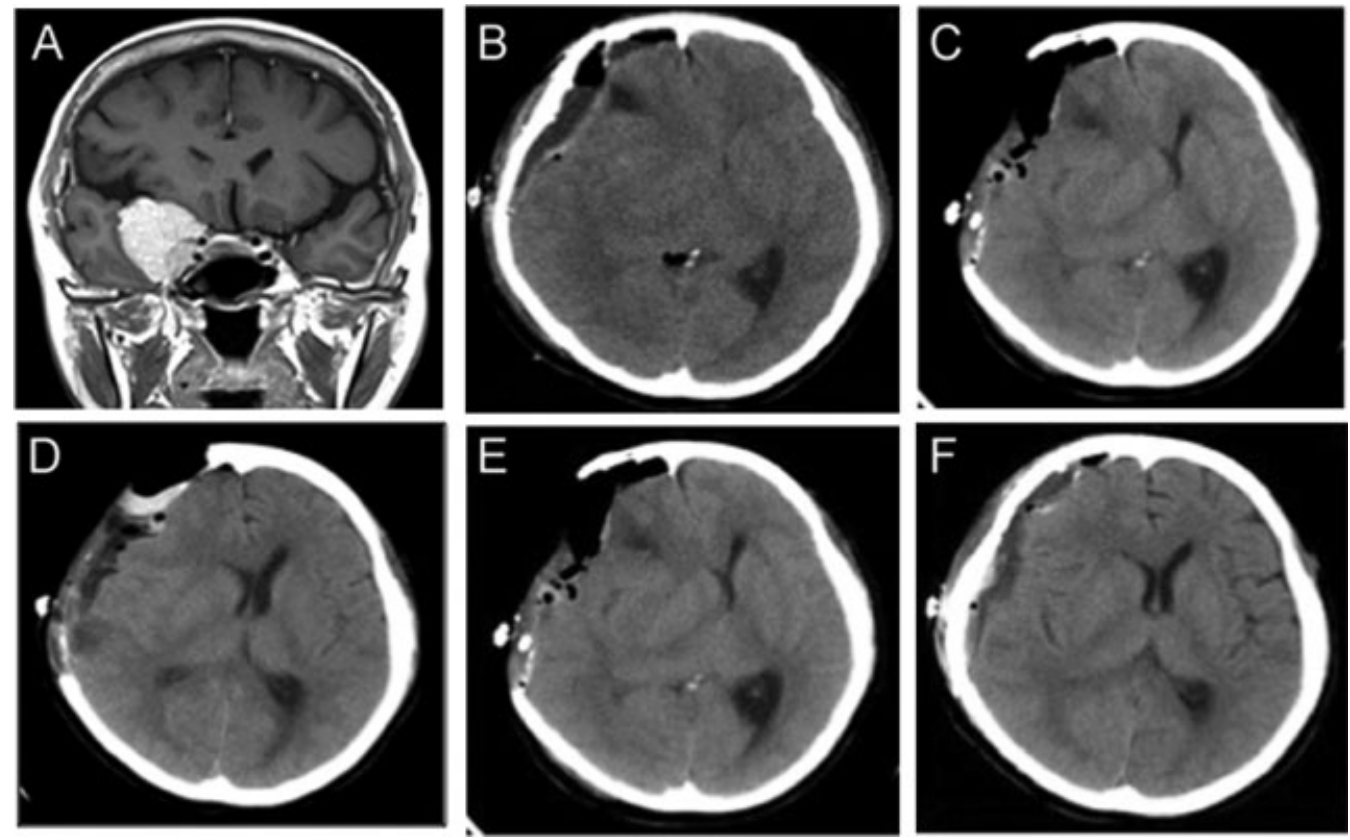

Fig. 1 Case 1. (A) Preoperative T1-weighted postcontrast magnetic resonance image. (B-F) Postoperative computed tomography (CT) scans. (A) Coronal image showing a spheno-orbital meningioma. (B) CT image on postoperative day 2 showing a midline shift and fluid collection in the epidural space. (C) Decompressive craniectomy and fluid evacuation worsened the midline shift. (D) Bedridden management improved the patient's consciousness and the shift. (E) Repeated midline shift was observed immediately after she started to walk. (F) Epidural blood patch and cranioplasty dramatically improved the midline shift and her consciousness. 

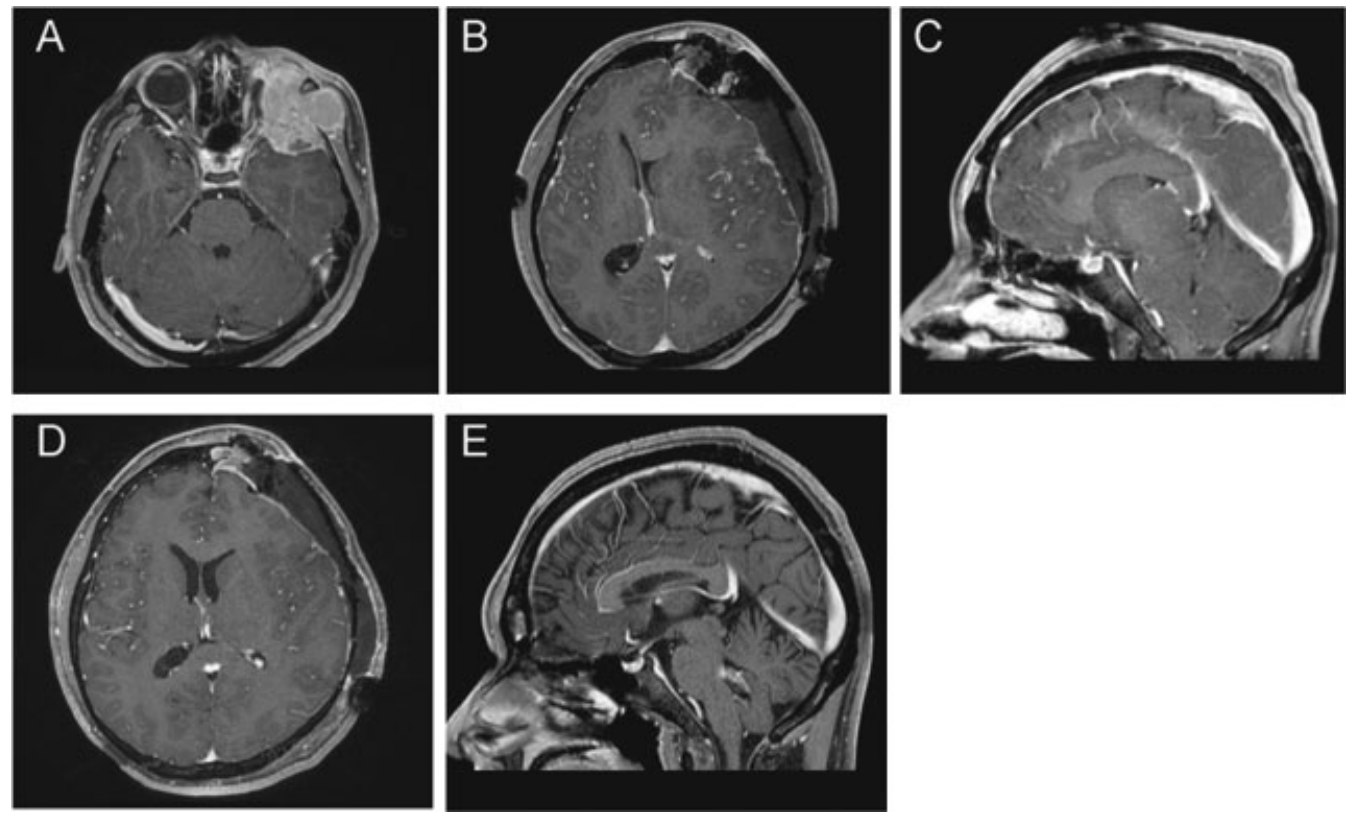

Fig. 2 Case 2. (A) Preoperative, (B, C) post-craniotomy, and (D, E) after epidural blood patch (EBP) T1-weighted postcontrast magnetic resonance images. (A) Axial image showing bone-destructive mass invading the left orbit and the middle fossa. Left eyeball was already enucleated to treat retinoblastoma at age 10 months. (B) Axial image demonstrating a severe midline shift and epidural fluid collection. (C) Sagittal image showing posterior fossa crowding with pontine flattening and tonsil invagination, or "sag," into the foramen magnum. (D) Axial and (E) sagittal images 4 days after EBP showing rapid improvement of the midline shift, tonsillar sag, and cisternal obliteration.

day. Follow-up magnetic resonance imaging (MRI) studies demonstrated the total resection of the tumor. However, the midline shift, tonsillar sag into the foramen magnum, and prepontine cistern obliteration due to brainstem swelling (-Fig. 1B, C) were compatible with intracranial hypotension. Because the CSF leak from the LD inserted site was strongly suspected, $40 \mathrm{~mL}$ of EBP was placed at L3-L4 on postoperative day 15 , and his postural headache disappeared immediately and completely. Neuroimages 4 days after the EBP ( - Fig. 2D, E) showed immediate improvement of the midline shift, tonsillar sag, and obliteration of the cisternal space in the posterior fossa.

\section{Discussion}

To our knowledge, this is the first report of two patients with IH after LD-assisted skull base surgery to increase recognition of this syndrome. Although many LD-induced IH cases after supratentorial clipping surgery have been reported, ${ }^{1-5}$ neurosurgeons' knowledge about this entity is still poor, and an accurate diagnosis and adequate treatment is not always performed as in our first case. Our report should promote awareness of this rare but potentially critical condition not only in LD-supported vascular surgery but also in skull base surgery.

As mentioned, all the previously reported $\mathrm{IH}$ cases after LD were craniotomies for supratentorial ruptured ${ }^{1-3,5}$ and unruptured $^{1}$ aneurysms, except one case of clival chordoma resected with LD. ${ }^{4}$ The reasons why the past cases centered on clipping surgeries was not adequately explained. To begin with, recognition of this condition by skull base surgeons is too absent to include this entity in the differential diagnosis for postoperative postural headache or neurologic deterioration. Second, compared with aneurysmal surgery, it is common for patients who undergo resection of a skull base tumor to be at bed rest for a prolonged time that obscures the postural headache due to the potential IH. Because bed rest management and the Trendelenburg position are conservative treatment for $\mathrm{IH}$, an early diagnosis may be difficult in those situations. Komotar et al reported ${ }^{2}$ that this syndrome is most evident in patients who are relatively neurologically intact, which supports our speculation. Third, although the accurate incidence rate of IH following both types of surgeries is unknown, the overall number of aneurysmal surgeries is likely to be much than that of skull base surgery, and therefore patients with $\mathrm{IH}$ are much more likely in LD-supported clipping surgery.

The mechanism why IH occurs after craniotomy with LD has not been well elucidated. It is well known that LD significantly increases the risk of $\mathrm{IH}$. Although the past reports about IH in aneurysmal surgery suspect the fenestration of the lamina terminalis and third ventriculostomy may alter CSF flow dynamics and increase the probability of this condition, ${ }^{2}$ these procedures were not performed in our cases. Additionally, compared with our cases, IH following clipping surgery tends to occur in much earlier postoperatively with a mean IH presentation of 45.8 hours postoperatively. $^{2}$ In the previous reports analyzing risk factors for post-LP headache, younger age, between 20 and 40 years (or 30 and 50), and female gender are reported to be definitive risk factors. ${ }^{6,7}$ Our two patients (a 57-year-old woman and 31-year-old man) did not fully match these factors. These 
clinical differences suggest a different pathophysiology for IH after LD-assisted skull base surgery. First, although the precise identification of the CSF leak site after LD removal is difficult, a persistent CSF leak through the LD-inserted pinhole is mandatory to develop this condition. It is well accepted that the total amount of the intraoperative CSF removal via LD is not related to IH development because a greater amount of CSF is continuously generated. Second, continuous CSF leakage may occur due to an insufficient or delayed healing process of the dural pinhole at the spinal drain inserted site. The dural pinhole is thought to be closed or blocked by the surrounding epidural fat tissue or the small amount of hematoma derived from the injured dural venous plexus. Therefore it is speculated that those patients with a low BMI (like our case 1) or whose healing process is poor or late are likely to be at greater risk for developing $\mathrm{IH}$. We also suspect the multiple attempts to insert the $L D$, as well as the size of the needle and drain tube itself, may increase the risk of $\mathrm{IH}$ development. Finally, unilateral broad dissection of the skull base induces the detachment of the dura from the bony base that may worsen the midline shift concomitant with $\mathrm{IH}$. Either frontal or temporal base dissection is insufficient to induce the severe midline shift. Both of them, sustained CSF leakage and unilateral broad dural dissection from the skull base, are needed for the presentation of $\mathrm{IH}$ and subsequent severe midline shift after skull base surgery.

Although the underlying mechanism for $\mathrm{IH}$ after LD-assisted skull base surgery may be different from that of aneurysmal surgery, the same treatment strategy provides a favorable outcome. The conservative treatment including bedrest and the Trendelenburg position is generally recommended because they can resolve mild IH completely. ${ }^{2}$ For severe IH patients with continuous postural headache refractory to conservative therapy, however, EBP provides immediate improvement in both clinical symptoms and neuroimaging findings. ${ }^{1,4,5}$ The most important point for the neurosurgical community is to include IH as a differential diagnosis in not only clipping but also skull base surgery, especially when LD assisted, and not to try to evacuate the epidural collection that leads to further neurologic deterioration and even lethality. ${ }^{4}$ However, midline shift in neuroimages often makes it difficult to distinguish postoperative brain edema from $\mathrm{IH}$.

This report has several limitations due to the small number of the cases. Furthermore, our presumed risk factors involved in IH development-low BMI, the delayed healing process, and multiple attempts at insertion-must be validated in a larger case series.

\section{Conclusion}

IH following LD-assisted skull base surgery with broad bony and dural dissection is life threatening but difficult to diagnose in the early stages. EBP provides a favorable outcome immediately and completely. All skull base surgeons must become aware of this condition. Future studies are needed to clarify the risk factors associated with the development of IH following LD-assisted skull base surgery.

\section{References}

1 Samadani U, Huang JH, Baranov D, Zager EL, Grady MS. Intracranial hypotension after intraoperative lumbar cerebrospinal fluid drainage. Neurosurgery 2003;52(1):148-151; discussion 151152

2 Komotar RJ, Mocco J, Ransom ER, et al. Herniation secondary to critical postcraniotomy cerebrospinal fluid hypovolemia. Neurosurgery 2005;57(2):286-292; discussion 286-292

3 Bloch J, Regli L. Brain stem and cerebellar dysfunction after lumbar spinal fluid drainage: case report. J Neurol Neurosurg Psychiatry 2003;74(7):992-994

4 Loya JJ, Mindea SA, Yu H, Venkatasubramanian C, Chang SD, Burns TC. Intracranial hypotension producing reversible coma: a systematic review, including three new cases. J Neurosurg 2012; 117(3):615-628

5 Mathew L, Komotar R. Epidural blood patch for severe postoperative intracranial hypotension. J Neurosurg Anesthesiol 2008; 20(1):49-52

6 Evans RW, Armon C, Frohman EM, Goodin DS. Assessment: prevention of post-lumbar puncture headaches: report of the therapeutics and technology assessment subcommittee of the American Academy of Neurology. Neurology 2000;55(7):909-914

7 Amorim JA, Gomes de Barros MV, Valença MM. Post-dural (postlumbar) puncture headache: risk factors and clinical features. Cephalalgia 2012;32(12):916-923 